\title{
Outcome of Patients with Diabetic Ketoacidosis in South of Iran
}

\author{
Hossein Moravej ${ }^{1,2}$, Homa Ilkhanipour $^{2}$, Zahra seratishirazi ${ }^{2}$, Maryam Sadat Azargoon $^{3}$ and Anis Amirhakimi ${ }^{2}$ \\ ${ }^{1}$ M.D. Neonatal Research Center, Shiraz University of Medical Sciences, Shiraz, Iran. \\ ${ }^{2}$ M.D. Pediatric Endocrinology Department, School of Medicine, Shiraz University of Medical Sciences, Shiraz, Iran. \\ ${ }^{3}$ M.D. School of Medicine, Shiraz University of Medical Sciences, Shiraz, Iran.
}

\begin{abstract}
Received: : June 16, 2017; Accepted: June 30, 2017; Published: July 17, 2017
*Corresponding author: Homa Ilkhanipour.M.D, Postal address: Department of Pediatrics, Namazi Hospital, Shiraz, Iran; Tel: 09173137803; Fax: 07136474298;E-mail: H_ilkhani66@yahoo.com
\end{abstract}

\begin{abstract}
Introduction: Diabetic Keto acidosis (DKA) is the most common cause of mortality in children with diabetes mellitus. The aim of this study is to investigate the complications of DKA and its complications in hospitalized patients in Namazi hospital, Shiraz, Iran.

Methods: This study is a sectional descriptive survey. The statistical population included patients with DKA in the age range of 1 month to 18 years, who were hospitalized in Nemazee hospital in 2009 to 2013. All patients' records were investigated to find out if there were complications of DKA. Data analysis was conducted using SPSS software.

Results: Total population of patients was 224 cases $59.8 \%$ of patients had newly diagnosed diabetes. The cause of DKA in the other $40.2 \%$ of patients who were known cases of diabetes included lack of insulin injection in $16.5 \%$, infection in $13.4 \%$, stress of surgery or trauma in $0.9 \%$, and unknown cause in $9.4 \%$ of the cases. The common complications included renal failure in 15 cases, coma in 2 cases, cerebral edema in 2 cases, cardiac arrhythmia in 3 cases, infection in 10 cases, and mortality in 4 cases. The mortality rate was $1.7 \%$. There was a significant relation between mortality and low level of consciousness at admission time, hospitalization in ICU, hypokalemia during treatment, and older age.
\end{abstract}

Conclusions: The cause of DKA was dominantly unknown. Renal failure was considered as the dominant complication in this hospital. Since there was $1.7 \%$ mortality rate of DKA in this hospital, there was an urgent need for accurate and correct treatment.

Keywords: Diabetic ketoacidosis; mortality; complication; type 1 diabetes mellitus

\section{Introduction}

Diabetes is a metabolic disease characterized by increased blood glucose resulting from defects in insulin secretion, insulin function, or both of them. [1]

During the past decades, frequency of type 1 diabetes has increased. On average, 78,000 diabetic children are discovered each year. [2] One of the most common initial presentations of diabetes is DKA. DKA is the most important cause of mortality in children with type 1 diabetes. . DKA is a metabolic derangement which consists of a triad of hyperglycemia, metabolic acidosis and ketosis. It occurs due to a severe deficiency in insulin, or an increase in counter regulatory hormones. Risk factors for progression of DKA include previous infectious diseases such as, pneumonia and urinary tract infection, ischemic events such as heart attacks and strokes, pancreatitis, cessation of treatment with insulin, non-compliance with the rules of the medication, poor education for the patient concerning the management of diabetes, intentional cessation of insulin, and use of drugs which affect the metabolism of carbohydrates. [3]

In a systematic review 65 cohort studies on 29,000 children with diabetes in 31 institutions showed that DKA as the initial manifestation of type 1 diabetes varied from $12.8 \%$ to $80 \%$. The highest frequencies were found in Saudi Arabia, United Arab Emirates, and Romania. The least frequencies were found in Canada, Sweden and Slovak Republic. [4]

The primary goals in the treatment of these patients are as follow:

- Dehydration treatment with intravenous fluids

- Correction of glucose and ketoacidosis using insulin

- Correction of electrolytes

- Treatment of underlying factors such as infection and ischemia. [3]

DKA has many complications. It is the most common cause of mortality in children with diabetes. [2]

Recent data found in developing countries shows that the most important causes of mortality in patients with DKA are shock, sepsis, cerebral edema, and renal failure. [5]

A study in India showed that delay in diagnosis is the most important risk factor for mortality in DKA patients. [5]

Generally, mortality rate in children with DKA is between 0.15 to 0.35 in the developed countries, such as Canada, the United States and the United Kingdom. [6-7] this rate is between $3.4 \%$ and $13.4 \%$ in developing countries, such as India, Pakistan and Bangladesh. [8-12] 
Cerebral edema is the most important cause of mortality. [13] Prevalence of cerebral edema is reported between 0 to 5.5 percent in the developed countries; however, this rate is between 25 and 26 percent in developing countries. [2]

The real cause of cerebral edema is still unknown. [14] It is controversial whether hypoperfusion and ischemia lead to cytotoxic edema, or reperfusion and hyperper fusion lead to vasogenic edema. [2]

A cohort study showed that the prevalence of cerebral edema in children with DKA, who were hospitalized in PICU, was about $26 \%$. The most important factor associated with cerebral edema was the high volume of primary Bolus fluid. [15]

Low amount of fluid therapy in patients with shock and DKA may increase the risk of renal failure and cerebral edema. Sepsis is another cause of mortality in children with DKA. Sepsis not only results in DKA, but also increases the risk of renal failure and mortality in DKA patients. [2]

A study reported that among 63 children with DKA, 13 cases suffered from infections. These infections mostly included upper respiratory infection, pneumonia and diarrhea. [16]

In a study conducted $58 \%$ of patients had sepsis. But only in one-fifth of them a focus of infection was identified. [15]

Shock as a presentation in DKA is rare in the literature of developing countries. [2]

Renal failure as a complication of DKA is rare in the developed countries. [17] A study in Bangladesh showed that the prevalence of renal failure in patients with DKA was about $3.7 \%$. [8]

In a study in India, the prevalence of acute renal failure in patients with DKA was reported as $11.5 \%$. Furthermore, mortality of the patients suffering from renal failure was between $40 \%$ and $72 \%$. [17]

Severe dehydration in children with low fluid intake and delay in diagnosis of DKA may lead to renal failure in DKA. [18]

Hypokalemia is another complication of DKA. [19] In a study conducted on 54 patients with DKA, $5.6 \%$ of the patients suffered from Hypokalemia. [20]

A cohort study in which they investigated the outcome of septic patients with DKA in Taiwan during 2004 to 2013. Results showed that among 403 patients with DKA, 144 cases suffered from infection, mostly the respiratory tract one. Urinary tract infection, intra-abdominal infections, skin and soft tissue infections were reported as the dominant ones, respectively. Negative gram microorganisms were the most dominant microorganisms. [21]

A study showed that use of standardized diabetic ketoacidosis treatment protocol improved clinical outcome of the patients and decreased length of hospitalization of these patients. [22]

Given the importance of DKA as the leading cause of death in children with diabetes, and the small number of studies in this field in Iran, the present study aimed at investigating the prevalence of diabetic ketoacidosis consequences and the complications in patients with DKA hospitalized in Nemazee hospital, Shiraz, Iran, during 2009 to 2013.

\section{Methods}

This study was a sectional descriptive survey. The statistical population consisted of patients in the age range of 1 month to 18 years with a definite diagnosis of DKA, who were hospitalized in Nemaazi hospital, the referral center in the south of Iran, since 2009 to 2013.

Documents of all patients were investigated. All reports with DKA diagnosis were investigated again. Those patients with DKA having venous ph $<7.3$, blood sugar $>250 \mathrm{mg} / \mathrm{dl}$ and bicarbonate $<15$ and positive ketonuria were included in the study. Patients who had just hyperglycemia or incomplete records were excluded.

The required information of patients was recorded in special forms. The forms contained information on patients' gender, age, level of consciousness, time of admission, transfer to pediatric intensive care unit, duration of their hospitalization, their recovery time, blood culture, urine culture, CRP, ESR, WBC, blood sugar, creatinine, blood urea nitrogen, potassium, sodium, $\mathrm{HCO}$, and PH tests.

Resolution of DKA was considered when the consciousness was normal, there was no vomiting, patient $\mathrm{PH}$ was more than 7.3 , and serum bicarbonate level was more than 15 .

Complications, including death, coma, cerebral edema, cardiac arrhythmia, hypoglycemia, hypokalemia, hypernatremia, infection, and renal failure were recorded on the special form. Potassium less than $3.5 \mathrm{meq} /$ lit was called hypokalemia, and the corrected sodium more than 150 meq / lit was called hypernatremia. Cerebral edema was recorded if irritability and decreased consciousness level were found clinical signs and symptoms of cerebral edema were mentioned, and the brain CT scan showed brain edema.

Data analysis was conducted using SPSS software, version 20 . The qualitative variables were compared using Chisquare and Fisher exact test, and the quantitative variables were compared using independent T-test. The $\mathrm{P}$ value $<0.05$ was selected to examine the significance of the results.

\section{Results}

In the present study, 224 patients with DKA in the age range of one month to 18 years (on average 13 years old) participated in the study. They consisted of 82 males (36.6\%) and 142 females (63.4\%).

Of the total patients, 41 cases $(18.3 \%)$ were hospitalized in pediatric intensive care unit. 158 patients (70.5\%) had started their treatment in Nemazee hospital from the beginning, and 66 cases $(29.5 \%)$ had started their treatment at other medical 
centers, and then they were referred to Nemazee hospital.

113 cases $(50.4 \%)$ were in normal consciousness level, 103 ones $(46 \%)$ were sleepy and 8 ones $(3.6 \%)$ were in coma status on their arrival.

In our study, 134 patients $(59.8 \%)$ were new case of diabetes, and 90 patients $(40.2 \%)$ were known case of diabetes. Mortality occurred in 4 patients $(1.78 \%)$. Two patients $(0.89 \%)$ suffered from cerebral edema, 3 patients (1.33\%) suffered from cardiac arrhythmia, 15 patients (6.69\%) suffered from renal failure, and 10 ones (4.46\%) suffered from infection.

DKA was the initial manifestation of diabetes in 59.8\% of the patients. In the rest of them the underlying cause of DKA was lack of insulin injection (16.5\%), infection (13.4\%), stress of surgery and trauma (0.9\%), and unknown cause $(9.4 \%)$.

During 2009 to 2013, number of the recorded DKA gradually increased. The lowest percentage $(9.37 \%)$ of all DKA cases was seen in 2009 , and the highest percentage (28.6\%) was recorded in 2013.

Concerning the duration of hospitalization, 79 cases $(35.3 \%)$ were hospitalized for 2 days. The duration of hospitalization varied from 1 day to 44 days.

Concerning the resolution time of patients with DKA, the most frequent time was less than 12 hours (66.6\%). Then, it was $13-24$ hours (28.6\%), 25-36 hours (2.34\%), 37-48 hours $(1.87 \%)$, and more than 48 hours $(0.47 \%)$, respectively.

Among 224 patients in this study, just three cases received bicarbonate during their treatment. There was a significant relationship between bicarbonate receiving and admittance in the ICU (P-value less than 0.05). In fact, patients who were hospitalized in PICU received bicarbonate more than other patients did. However, there was not a significant relation between $\mathrm{PH}$ value and bicarbonate receiving.

(Table 1) shows the relationship between the resolution of DKA in patients and different variables. As it is evident, there is a significant relationship between the level of consciousness of the patients at the time of arrival to the emergency center and the resolution time of DKA.

Independent $\mathrm{T}$ test was used to compare the quantitative variables in two levels, and ANOVA test was used to compare more than two levels.

The relationships between withdrawal of DKA after the treatment and different variables, such as age, number of DKA attacks, duration of DKA symptoms before entering the hospital, $\mathrm{PH}$ and $\mathrm{HCO} 3$, were investigated. There was a positive correlation between withdrawal of DKA after the treatment and duration of having DKA symptoms before entering the hospital. Furthermore, there was a negative correlation between withdrawal of DKA after treatment and bicarbonate value. In other words, the withdrawal time was longer for those for whom DKA treatment was delayed. Moreover, the withdrawal time was longer for those who initially had lower bicarbonate levels.

\begin{tabular}{|c|c|c|c|c|}
\hline \multirow{2}{*}{\multicolumn{2}{|c|}{$\begin{array}{c}\text { Variable } \\
\text { Yes }\end{array}$}} & \multicolumn{2}{|c|}{ Mortality } & \multirow[t]{2}{*}{$P$ value } \\
\hline & & \multirow{2}{*}{\begin{tabular}{|c|} 
No \\
2
\end{tabular}} & \multirow[b]{2}{*}{80} & \\
\hline & Male & & & \multirow[b]{2}{*}{0.625} \\
\hline Sex & Female & 2 & 140 & \\
\hline \multirow[b]{2}{*}{$\begin{array}{c}\text { Time of diabetes } \\
\text { diagnosis }\end{array}$} & New case & 4 & 130 & \multirow[b]{2}{*}{0.151} \\
\hline & Known case & 0 & 90 & \\
\hline \multirow[b]{2}{*}{ First DKA } & Yes & 31 & 189 & \multirow[b]{2}{*}{1} \\
\hline & No & 4 & 0 & \\
\hline \multirow{3}{*}{$\begin{array}{c}\text { Level of } \\
\text { consciousness on } \\
\text { arrival to } \\
\text { emergency room }\end{array}$} & Alert & 0 & 113 & \multirow{3}{*}{0.000} \\
\hline & Drowsy & 0 & 103 & \\
\hline & Coma & 4 & 4 & \\
\hline \multirow[b]{2}{*}{$\begin{array}{l}\text { Admittance to } \\
\text { ICU }\end{array}$} & Yes & 3 & 38 & \multirow[b]{2}{*}{0.020} \\
\hline & No & 1 & 182 & \\
\hline \multirow[b]{2}{*}{$\begin{array}{c}\text { Cerebral edema } \\
\text { as a complication }\end{array}$} & Yes & 0 & 2 & \multirow[b]{2}{*}{1} \\
\hline & No & 4 & 218 & \\
\hline \multirow[b]{2}{*}{$\begin{array}{c}\text { Hypernatremia } \\
\text { during treatment }\end{array}$} & Yes & 1 & 18 & \multirow[b]{2}{*}{0.3} \\
\hline & No & 3 & 202 & \\
\hline \multirow[b]{2}{*}{$\begin{array}{c}\text { Hypokalemia } \\
\text { during treatment }\end{array}$} & Yes & 3 & 49 & \multirow[b]{2}{*}{0.04} \\
\hline & No & 1 & 171 & \\
\hline
\end{tabular}

Furthermore, the relation between mortality and different variables, such as gender, being a new case or being a known case, rank of DKA attack, the center in which they had received the initial treatment, causes of DKA, level of consciousness on the arrival in the emergency ward and ICU, cerebral edema as a complication, hypokalemia or hypernatremia during the treatment, age, $\mathrm{PH}$ value, $\mathrm{HCO}_{3}$ value, the duration of DKA symptoms, the number of attacks the patient suffered from, were investigated.

All values for quantitative variables were expressed as mean and standard deviation, and the qualitative variables are expressed in terms of percentage.

As it is evident in the table 1 , there is a significant relation between low levels of consciousness, coma and death. Admittance to ICU was associated with increased the risk of death. In addition, hypokalemia was effective in increasing the mortality.

The relation between cerebral edema and different variables was investigated. As can be seen in (Table 2), there is not significant relation between cerebral edema and other variables. 
Table 2: Correlation between cerebral edema and different variables

\begin{tabular}{|c|c|c|c|c|}
\hline \multirow{2}{*}{\multicolumn{2}{|c|}{ Variable }} & \multicolumn{2}{|c|}{$\begin{array}{l}\text { Occurrence of } \\
\text { cerebral edema }\end{array}$} & \multirow[t]{3}{*}{$P$ value } \\
\hline & & \multicolumn{2}{|l|}{ No } & \\
\hline & Male & 0 & 82 & \\
\hline Sex & Female & 2 & 140 & 0.53 \\
\hline \multirow{2}{*}{$\begin{array}{l}\text { Time of diabetes } \\
\text { diagnosis }\end{array}$} & New case & 2 & 132 & \multirow[b]{2}{*}{0.35} \\
\hline & Known case & 0 & 90 & \\
\hline \multirow[b]{2}{*}{ First DKA } & Yes & 2 & 191 & \multirow[b]{2}{*}{1} \\
\hline & No & 0 & 31 & \\
\hline \multirow{3}{*}{$\begin{array}{l}\text { Level of consciousness } \\
\text { on arrival to emergency } \\
\text { room }\end{array}$} & Alert & 0 & 113 & \multirow{3}{*}{0.28} \\
\hline & Drowsy & 2 & 101 & \\
\hline & Coma & 0 & 8 & \\
\hline \multirow[b]{2}{*}{ ICU Admittance } & Yes & 1 & 40 & \multirow[b]{2}{*}{0.33} \\
\hline & No & 1 & 182 & \\
\hline \multirow{2}{*}{$\begin{array}{l}\text { Mortality as a } \\
\text { complication }\end{array}$} & Yes & 0 & 4 & \multirow[b]{2}{*}{1} \\
\hline & No & 2 & 218 & \\
\hline \multirow{2}{*}{$\begin{array}{l}\text { Hypernatremia during } \\
\text { treatment }\end{array}$} & Yes & 0 & 19 & \multirow[b]{2}{*}{1} \\
\hline & No & 2 & 203 & \\
\hline \multirow{2}{*}{$\begin{array}{l}\text { Hypokalemia during } \\
\text { treatment }\end{array}$} & Yes & 0 & 52 & \multirow[b]{2}{*}{1} \\
\hline & No & 2 & 170 & \\
\hline
\end{tabular}

\section{Discussion}

In the present study, following results were obtained by evaluation of the complications of DKA in patients with diabetic ketoacidosis in Nemaazi hospital in the south of Iran, during a five-year period.

The present study showed that the prevalence of DKA has been gradually increasing during a five-year period.

As 3\% increase in the number of diabetic patients in the United States is recorded, the following findings are not unexpected. [23]

134 patients (59.8\%) participated in the present study were new cases of diabetes, and 90 cases were known cases of diabetes. [4] The prevalence of diabetic ketoacidosis as the first manifestation of diabetes varied from $12.8 \%$ to $80 \%$. The dominant frequency was found in the developing countries. The lowest frequency was found in Sweden and Canada (4). It shows that education on the early symptoms of diabetes should be enhanced, so that new diabetic patients are not neglected, and they would not refer to hospitals having severe complications such as DKA.

Lack of insulin injection and infection are the most causes of DKA among all known cases.

[24] Lack of insulin injection was considered as the most important cause of DKA in 52\% of cases. [24] This difference may be due to differences in the populations studied and the increased sensitivity and awareness of parents about the importance of insulin injection in diabetic children.
In this study, it was found that $70.5 \%$ of patients were hospitalized in a specialized center from the beginning of their treatment. The remaining $29.5 \%$ of the cases started their treatment in other medical centers. Investigating the relationship between DKA withdrawal time after the treatment and different variables showed that DKA patients who were initially treated in Nemaazi hospital were withdrawn sooner than patients who started their treatment in other medical centers. Therefore, it seems that it is necessary to train all physicians and medical staffs in hospitals on the proper treatment of these patients.

Mortality occurred in 4 patients $(1.78 \%)$. The mortality rate in DKA is between $0.15 \%$ and $0.35 \%$ in the developed countries, such as the United States of America, Canada and the UK (6-7). This rate is between $3.4 \%$ and $13.4 \%$ in the countries such as India, Pakistan and Bangladesh (8-11). These differences may be due to the differences in the duration of feeling bad before referring to a hospital, the improper treatments, and the differences in the medical care received by patients with DKA. The mortality rate in the present study was higher than countries such as USA, Canada and the UK but was lower than countries, such as India and Bangladesh. Therefore, it seems that we need more investigations about the causes of this mortality rate and the ways for its reduction.

Among 224 patients in our study, only 3 cases (1.33\%) received bicarbonate. However, this figure is consistent with the recent guidelines, which shows that bicarbonate indications are very limited in the treatment of patients with DKA.

There was a significant relationship between the withdrawal time of patients with DKA and their level of consciousness. In other words, the lower is the patient's level of consciousness; the longer is the healing period. Of course, this finding is not unexpected. The more is the severity of dehydration and the duration of DKA, the lower is the patient's level of consciousness.

The mortality rate was higher in patients who were in the coma status at the time of admission to the emergency ward. It may indicate that the delay in delivering the patients to the hospital is associated with the risk of mortality in patient with DKA.

The prevalence of cerebral edema in patients was $89 \%$. The cerebral edema was the most important cause of mortality in patients with DKA. [15] reported that cerebral edema in children with DKA who were admitted to the intensive care unit of children in North India was about 26\%. According to this study, the causes of cerebral edema were high fluid intake and respiratory failure requiring ventilation in the ICU. [15] Although the main part of the present study focused on children hospitalized in pediatric endocrinology ward, and did not require hospitalization in ICU, the mentioned cohort study was conducted in India and focused on children hospitalized in ICU.

In our study, 15 patients (6.69\%) suffered from renal failure; however, renal failure in patients with DKA was not a prevalent complication in the developed countries. [17] in Iran, $4.7 \%$ of patients with DKA were suffering from acute renal failure. [16] reported $3.7 \%$ incidence of renal failure in patients with DKA in Bangladesh. [8]

If children with DKA suffer from renal failure, mortality rate will be in the range of 40 to $72 \%$. [2] 
Sepsis shock and rhabdomyolysis are among the causes of kidney failure. Treatment is very difficult when the patient suffers from renal failure. Oliguria and anuria are not reliable criteria for renal failure diagnosis. Furthermore, urea and creatinine levels may be increased in DKA. Fluid restriction, sepsis, shock (hypovolemic or septic) and fear of suffering from cerebral edema can increase the risk of renal failure. Severe dehydration and the delay in the diagnosis of DKA may lead to acute tubular necrosis. [2]

Treatment of renal failure accompanied by DKA creates some problems for physicians. Peritoneal dialysis may even lead to drastic changes in the patient's blood sugar.

There is no standard guideline for the treatment of renal failure in patients with DKA at the present time. Renal failure may be due to delay in diagnosis and delay in admittance of patients with DKA to the hospital. Of course, all aspects should be considered in the treatment to prevent renal failure. [2]

Hypokalemia was another complication that patients with DKA suffered from. In this study, hypokalemia increased the risk of mortality during the treatment.

Determined Hypokalemia in 54 patients with DKA. $5.6 \%$ of their patients suffered from hypokalemia. However, in the present study, $23.2 \%$ of the patients suffered from hypokalemia. [20] This difference may be due to differences in the type of populations under study. Cardiac arrhythmia was the most common complication in patients with DKA in adolescents and adults; however, contrary to our findings, cerebral edema was not a common complication in patients with DKA. [24] This difference may be due to differences in the age range of the populations under study.

Assessed the clinical outcomes in patients with DKA in Taiwan. The results showed that $35.7 \%$ of patients suffered from infection; however, $13.4 \%$ of patients suffered from infection in the present study. [21]

\section{Conclusion}

The present study showed that mortality of DKA in Nemaazi hospital in south of Iran is more than those reported from countries such as USA and Sweden. The mortality rate was higher in patients who were in the coma status at the time of admission to the emergency ward. Therefore, it seems that it is necessary to educate all family physicians, diabetic patients' parents, people and medical staffs about clinical manifestations of DKA for early diagnosis and referring DKA patients to hospital. Regarding to prevalence of complications such as renal failure and hypokalemia in our study it seems that DKA management guidelines and medical cares in our center needs more investigation and revision

\section{Acknowledgements}

The present study was financially supported by Shiraz University of Medical Sciences and approved with code no. 9301-01-7148. The study was extracted from the thesis written by Maryam Sadat Azargoon for M.D. degree. The authors would like to thank Dr. Nasrin Shokrpour and the center for development of clinical research of Nemazee Hospital in Shiraz University of medical Sciences for editorial assistance.

\section{References}

1. Long AE, Bingley PJ. The epidemiology of childhood diabetes. Europ J Ped. 2009; 19(7):304-308.

2. Poovazhagi V. Risk factors for mortality in children with diabetic ketoacidosis from developing countries. World J Diabetes. 2014;5(6):932938. Doi: $10.4239 /$ wjd.v5.i6.932

3. Maletkvoic J, Drexler A. Diabetic ketoacidosis and hyperglycemic hyperosmolar state. Endocrinol Metab clin North Am. 2013; 42(4):677695. Doi: 10.1016/j.ecl.2013.07.001

4. Usher- smith JA, Thompson M, Ercole A, Walter FM. Variation between countries in the frequency of diabetic ketoacidosis at first presentation of type 1 diabetes in children: a systematic review. Diabetologia. 2012; 55(11):2878-2894. Doi: 10.1007/s00125-012-2690-2

5. Poovazhagi V. Saradha S. Delayed diagnosis of Diabetic ketoacidosis in children- a cause for concern. Diabetes dev ctries. 2015;35(2):66-70. Doi: 10.1007/s13410-014-0245-4

6. Curtis JR, To T, Muirhead S, Cummings E, Daneman D. Recent trends in hospitalization for diabetic ketoacidosis in ontario children. Diabetes care. 2002;25(9):1591-1596.

7. Lawrence SE, Cummings EA, Caboury T, Daneman D. Population-based study of incidence and risk factors for cerebral edema in pediatric diabetic ketoacidosis. J Pediatr. 2005;146(50):688-692. Doi: 10.1016/j. jpeds.2004.12.041

8. Zabeen B. Nahar J, Mohsin F, Kishwar Azad, Nazmun Nahar. DKA in children-An experience in a tertiary hospital. Ibrahim Medical coll. J. 2008;2(1):17-20.

9. Lone SW, Siddiqui EU, Muhammed F, Atta I, Ibrahim MN, Raza J. Frequency, clinical characteristics and outcome of diabetic ketoacidosis in children with type-1 diabetes at a tertiary care hospital. J Pak Med Assoc. 2010; 60(9):725-729.

10. Jayashree M, Singhi S. Diabetic ketoacidosis: predictors of outcome in a pediatric intensive care unit of a developing country. Pediatr crit care Med. 2004; 5(5):427-433.

11. Kanwal SK, Band. A, Kumar V. Clinical profile of diabetic ketoacidosis in Indian children. Indian J Pediatr. 2012; 79(7):901-904. Doi: 10.1007/s12098-011-0634-3

12. Syed M, Kawaja FB, Saleem T, Khalid U, Rashid A, Humayun KN. Clinical profile and outcomes of pediatric patients with diabetic ketoacidosis at a tertiary care hospital in Pakistan. J Pak Med Assoc. 2011;61(11):1082-1087.

13. Mozzillo E, d'amico A, Factorusso V, Carotenuto B.b, Buono P.a, De Nitto E,et al. Cerebral accidents in pediatric diabetic ketoacidosis: Different complications and different evolutions. Horm Res pediatr. 2015; 84(2):139-144.

14. Hsia DS, Tarai SG, Alimi A, Coss-Bu JA, Haymond MW. Fluid Management in pediatric patients with DKA and rates of suspected clinical 
cerebral edema. Pediatr Diabetes. 2015;16(5):338-344. Doi: 10.1111/ pedi.12268

15. Tiwari LK, Jayashree M, Singhi S. Risk factors for cerebral edema in diabetic ketoacidosis in a developing country:role of fluid refractory shock. Pediatr crit care Med. 2012;13(2):e91-96. Doi: 10.1097/ PCC.0b013e3182196c6d

16. ASL AS, Maleknejad S, Kelachaye ME. Diabetic ketoacidosis and its complications among children. Acta Med Iran. 2011; 49(2):113-114.

17. Rosenbloom AL. Hyperglycemic crises and their complications in children. J Pediatr Endocrinol Metab. 2007;20(1):5-18. Doi: doi org/10.1515/JPEM.2007.20.1.5

18. Poovazhagi V, Senguttuvan P, Padmaraj R. Outcome of acute renal failure in children with diabetic ketoacidosis (DKA). Pediatric oncall. 2011;8(8):63-65.

19. Davis SM, Maddux AB, Alonso GT, Okada CR, Mourani PM, Maahscet DM. Profound Hypokalemia associated with severe diabetic ketoacidosis. Pediatr Diabetes. 2016;17(1):61-65.
20. Arora S, Cheng D, Wyler B, Menchine M. Prevalence of hypokalemia in ED patients with diabetic ketoacidosis. American J Med. 2012; 30(3):481-484

21. Cheng YC, Huang CH, Lin WR, Lu PL, Chang K, Tsai JJ, et al. Clinical outcomes of septic patients with diabetic ketoacidosis between 2004 and 2013 in a tertiary hospital in Taiwan. J Microbial Immunol Infect. 2016;49(5):663-671.

22. Thuzar M, Malabu UH, Tisdell B, Sangla KS. Use of standardised diabetic ketoacidosis management protocol improved clinical outcomes. Diabetes Res Clin Pract. 2014;104(1):e8-11. Doi: 10.1016/j.diabres.2014.01.016

23. Lawrence JM, Imperatore G, Dabelea D, Mayer-Davis EJ, Linder B, Saydah S, et al. Trends in incidence of type 1 diabetes among non-hispanic white youth in the U.S., 2002-2009. Diabetes. 2014;63(11):39383945. Doi: 10.2337/db13-1891

24. Mashayekhi M, Azimi R, Sadeghian B. Study of predisposing factors, complications and mortality of diabetic ketoacidosis in teens and adults. Iran J Med. 2001;8(23):63-70 (Persian) 